\title{
A Method for Selecting Optimal Number of Sensors to Improve the Credibility
}

\author{
Yi Chen, Jun Yu, Aixiang He, and Zhen-an Tang \\ Liaoning IC Technology Key Laboratory, School of Electronics Science \& Technology, \\ Dalian University of Technology, Dalian, Liaoning 116023, China
}

Correspondence should be addressed to Zhen-an Tang; tangza@dlut.edu.cn

Received 19 June 2015; Revised 25 September 2015; Accepted 29 September 2015

Academic Editor: Maria Luz Rodríguez-Méndez

Copyright (C) 2016 Yi Chen et al. This is an open access article distributed under the Creative Commons Attribution License, which permits unrestricted use, distribution, and reproduction in any medium, provided the original work is properly cited.

\begin{abstract}
With the development of sensors, it is possible to embed many sensors within a certain space, which makes the monitor and alarm system with multisensor possible. There are two important parameters in a monitor and alarm system, namely, the false alarm rate and the missed alarm rate. In this work, a method for selecting optimal number of sensors in the sensor array is presented to improve the credibility. The influence factors of the weights and the false alarm rate and the missed alarm rate of one sensor and total number of sensors are discussed. An experimental setup was developed. The monitoring methods of common strategies and the proposed optimal number of sensors strategy are compared graphically by the receiver operating characteristic curves and the area under receiver operating characteristic curve values. The receiver operating characteristic curves graphically prove that the optimal number of sensors' method presents the best performance, and it is shown that the optimal number of sensors' method has the highest area under receiver operating characteristic value (0.9631). This method may aid future users of the monitor and alarm system by providing an optimal number of sensors strategy for high credibility.
\end{abstract}

\section{Introduction}

With the development of sensor technologies, sensors have been improved with smaller size, lower power consumption, and better anti-interference ability, which makes it possible to embed many sensors within a certain local space. Multisensor information fusion pursues process redundant or complementary information from the multiple sources provided by the sensors to achieve results that are not feasible from a single sensor $[1,2]$. The monitor and alarm system (MAS) based on multisensor information fusion technologies has been widely used in many fields, such as the disease diagnosis $[3,4]$, the image fusion [1,5], the environment monitoring [6], and the security surveillance.

In security surveillance of high-risk industries, such as the traffic system [7], the vibration fault diagnosis [8], the recursive track [9], the medical surveillance [10], and the monitoring of hazardous materials [11], the credibility is one of the most important topics which attract extensive attention. A receiver operating characteristic (ROC) graph, which can combine FAR and MAR to one evaluation criteria, is a graphical plot that illustrates the performance of a binary classifier system as its discrimination threshold is varied [1214]. To compare the discrimination we may want to reduce the ROC performance to a single scalar value representing the expected performance. A common method is to calculate the area under the ROC curve (AUC) [15, 16]. A single performance indicator from the AUC can summarise the ROC curve as the higher the AUC value, the better the performance of the method. The credibility in the region of security surveillance means low false alarm rate (FAR) and low missed alarm rate (MAR) of the MAS [17]. The false alarm (FA) means that the MAS is triggered when it should not be triggered, while the missed alarm (MA) means that the MAS is not triggered when it should be triggered [18]. Smaller FAR or smaller MAR could be easily obtained by a sensor array and the proper tradeoff alarm strategy in an MAS. However, FAR and MAR are associated and it is difficult to simultaneously minimize the FAR and MAR in one MAS. To obtain small FAR and MAR simultaneously is the goal of high credibility MAS; thus the tradeoff between FAR and 
MAR is a fundamental problem which has attracted extensive interest by many researchers [19-23].

The FAR and MAR of an MAS are determined by the total number of sensors (TNS), the false alarm rate and the missed alarm rate of each sensor, and the alarm strategy. In this work, to make comprehensive estimation for the credibility of an MAS, the equivalent false alarm rate (EFAR), which reflects the FAR and MAR as well as the weights, is defined by the authors. The weights denote the loss of the FAR relative to the MAR. A quantitative method to select the optimal number of sensors (ONS) for minimizing the EFAR is given. The research results show that the proposed ONS strategy could improve the credibility when the TNS of the MAS is constant.

\section{The Method for Selecting Optimal Number of Sensors}

The parameters $p_{\mathrm{fa}}$ and $p_{\mathrm{ma}}$ are used to represent the false alarm rate and the missed alarm rate of a single sensor in the sensor array. The parameters $p_{\mathrm{fa}}$ and $p_{\mathrm{ma}}$ are equivalent to the false positive rate and one minus true positive rate, respectively, in ROC space. In a practical application, the values of $p_{\mathrm{fa}}$ and $p_{\mathrm{ma}}$ can be evaluated by the average value, where $p_{\mathrm{fa}}$ is the number of the negatives incorrectly classified divided by the total negatives and $p_{\mathrm{ma}}$ is the number of the positives incorrectly classified divided by the total positives. We propose an EFAR model with the following assumption.

The sum of $p_{\mathrm{ma}}$ and $p_{\mathrm{fa}}$ is less than 1 . The expression $1-p_{\text {ma }}$ (i.e., true positive rate in ROC space) represents the correct alarm decision rate. Therefore, the assumption demands that each sensor in the array produces useful information for detection. In ROC space, this assumption means that the (false positive rate and true positive rate) pairs should be in the upper triangular region, because the pairs in the diagonal represent the strategy of random guessing and in lower right triangle will perform even worse.

We may choose the alarm stratagem as follows: the result of the MAS is positive if $n$ sensors in the system give positive results. Here $n$ is the selected number of sensors (SNS). Assuming that the TNS in MAS is $M$, then $n$ is in the range from 1 to $M$. Obviously, $n=1$ corresponds to the lowest MAR but the highest FAR; and $n=M$ possesses the lowest FAR but the highest MAR. To study the optimized $n$ (ONS) of the system, the FAR and MAR associated with $n$ are derived from Bernoulli trials as follows:

$$
\begin{aligned}
\operatorname{FAR}[n] & =\sum_{j=n}^{M}\left(\begin{array}{c}
M \\
j
\end{array}\right) p_{\mathrm{fa}}^{j}\left(1-p_{\mathrm{fa}}\right)^{M-j}, \\
\operatorname{MAR}[n] & =\sum_{j=0}^{n-1}\left(\begin{array}{c}
M \\
j
\end{array}\right)\left(1-p_{\mathrm{ma}}\right)^{j} p_{\mathrm{ma}}^{M-j},
\end{aligned}
$$

where $\left(\begin{array}{c}M \\ j\end{array}\right)$ is the combination of selecting $j$ items from a set $M$. The FAR exists only when the output of the MAS is in alarm state; therefore, the expression $\operatorname{FAR}[n]$ is the sum of the false alarm rate with more than $n$ sensors being in alarm state. In contrast, the MAR exists only when the output of the MAS is in nonalarm state, and consequently the expression
$\operatorname{MAR}[n]$ denotes the sum of the missed alarm rate with less than $n$ sensors being in nonalarm state.

The objective function and constraints are as follows:

$$
\begin{array}{ll}
\operatorname{argmin} & \operatorname{EFAR}[n]=c_{\mathrm{fa}} \times \operatorname{FAR}[n]+c_{\mathrm{ma}} \times \operatorname{MAR}[n], \\
\text { s.t. } & \left\{\begin{array}{l}
1 \leq n \leq M \\
n \text { is an integer, }
\end{array}\right.
\end{array}
$$

where the coefficients $c_{\mathrm{fa}}$ and $c_{\text {ma }}$ denote the weight of FAR and MAR, respectively. The EFAR is the normalized rate of the FAR and MAR and $c_{\mathrm{fa}}$ is equal to $1-c_{\mathrm{ma}}$. Bigger $c_{\mathrm{fa}}$ shows that the FAR is more important than MAR in the monitoring strategy in the MAS.

The objective function and constraints are nonlinear programming with integer variables. In order to get the solution of the problem, a variable $k$ is introduced. If the index $k$ is the ONS of the MAS, then the range is from 1 to $M-1$ in the forward difference and from 2 to $M$ in the backward difference. A method based on recurrence relations of forward difference and backward difference is proposed for selecting the ONS. Suppose that $\operatorname{EFAR}(k)$ denotes the solution of (2), the expressions are as follows:

$$
\begin{array}{ll}
\operatorname{EFAR}(k+1)-\operatorname{EFAR}(k) \geq 0, & k \in[1, M-1], \\
\operatorname{EFAR}(k)-\operatorname{EFAR}(k-1) \leq 0, & k \in[2, M] .
\end{array}
$$

According to the first formula in (3), the expression can be changed into

$$
\begin{aligned}
& \operatorname{EFAR}(k+1)-\operatorname{EFAR}(k) \\
& =c_{\mathrm{ma}}\left(\begin{array}{c}
M \\
k
\end{array}\right)\left(1-p_{\mathrm{ma}}\right)^{k} p_{\mathrm{ma}}^{M-k} \\
& \quad-c_{\mathrm{fa}}\left(\begin{array}{c}
M \\
M-k
\end{array}\right)\left(1-p_{\mathrm{fa}}\right)^{M-k} p_{\mathrm{fa}}^{k} \geq 0 .
\end{aligned}
$$

As $\left(\begin{array}{c}M \\ k\end{array}\right)=\left(\begin{array}{c}M \\ M-k\end{array}\right),(4)$ can be changed into

$$
\frac{c_{\mathrm{ma}}\left(1-p_{\mathrm{ma}}\right)^{k} p_{\mathrm{ma}}^{M-k}}{c_{\mathrm{fa}} p_{\mathrm{fa}}^{k}\left(1-p_{\mathrm{fa}}\right)^{M-k}} \geq 1
$$

Further, (5) can be changed into

$$
\left(\frac{1-p_{\mathrm{ma}}}{p_{\mathrm{fa}}}\right)^{k}\left(\frac{p_{\mathrm{ma}}}{1-p_{\mathrm{fa}}}\right)^{M-k} \geq \frac{c_{\mathrm{fa}}}{c_{\mathrm{ma}}} .
$$

Taking natural logarithms on both sides, (6) can be changed into

$$
k \ln \frac{\left(1-p_{\mathrm{ma}}\right)\left(1-p_{\mathrm{fa}}\right)}{p_{\mathrm{ma}} p_{\mathrm{fa}}} \geq \ln \frac{c_{\mathrm{fa}}}{c_{\mathrm{ma}}}-M \ln \frac{p_{\mathrm{ma}}}{1-p_{\mathrm{fa}}} .
$$

According to the assumption, the sum of $p_{\mathrm{ma}}$ and $p_{\mathrm{fa}}$ is less than 1 . The expression $\left(1-p_{\mathrm{ma}}\right)\left(1-p_{\mathrm{fa}}\right) / p_{\mathrm{ma}} p_{\mathrm{fa}}>1$ and $\ln \left(\left(1-p_{\mathrm{ma}}\right)\left(1-p_{\mathrm{fa}}\right) / p_{\mathrm{ma}} p_{\mathrm{fa}}\right)>0$. 
Therefore, the result of forward difference is as follows:

$$
k \geq \frac{\ln \left(c_{\mathrm{fa}} / c_{\mathrm{ma}}\right)+M \ln \left(\left(1-p_{\mathrm{fa}}\right) / p_{\mathrm{ma}}\right)}{\ln \left(\left(1-p_{\mathrm{ma}}\right)\left(1-p_{\mathrm{fa}}\right) / p_{\mathrm{ma}} p_{\mathrm{fa}}\right)} .
$$

According to the second formula in (3), the expression can be changed into

$$
\begin{aligned}
& \operatorname{EFAR}(k)-\operatorname{EFAR}(k-1) \\
& =c_{\mathrm{ma}}\left(\begin{array}{c}
M \\
k-1
\end{array}\right)\left(1-p_{\mathrm{ma}}\right)^{k-1} p_{\mathrm{ma}}^{M-k+1} \\
& \quad-c_{\mathrm{fa}}\left(\begin{array}{c}
M \\
M-k+1
\end{array}\right)\left(1-p_{\mathrm{fa}}\right)^{M-k+1} p_{\mathrm{fa}}^{k-1} \leq 0 .
\end{aligned}
$$

As $\left(\begin{array}{c}M \\ k-1\end{array}\right)=\left(\begin{array}{c}M \\ M-k+1\end{array}\right),(9)$ can be changed into

$$
\frac{c_{\mathrm{ma}}\left(1-p_{\mathrm{ma}}\right)^{k-1} p_{\mathrm{ma}}^{M-k+1}}{c_{\mathrm{fa}} p_{\mathrm{fa}}^{k-1}\left(1-p_{\mathrm{fa}}\right)^{M-k+1}} \leq 1 .
$$

Further, (10) can be changed into

$$
\left(\frac{1-p_{\mathrm{ma}}}{p_{\mathrm{fa}}}\right)^{k-1}\left(\frac{p_{\mathrm{ma}}}{1-p_{\mathrm{fa}}}\right)^{M-k+1} \leq \frac{c_{\mathrm{fa}}}{c_{\mathrm{ma}}} .
$$

Taking the natural logarithms on both sides, (11) can be changed into

$$
\begin{gathered}
(k-1) \ln \frac{\left(1-p_{\mathrm{ma}}\right)\left(1-p_{\mathrm{fa}}\right)}{p_{\mathrm{ma}} p_{\mathrm{fa}}} \\
\leq \ln \frac{c_{\mathrm{fa}}}{c_{\mathrm{ma}}}-M \ln \frac{p_{\mathrm{ma}}}{1-p_{\mathrm{fa}}} .
\end{gathered}
$$

According to the assumption, the sum of $p_{\mathrm{ma}}$ and $p_{\mathrm{fa}}$ is less than 1 . The expression could be expressed as $\left(1-p_{\mathrm{ma}}\right)(1-$ $\left.p_{\mathrm{fa}}\right) / p_{\mathrm{ma}} p_{\mathrm{fa}}>1$ and $\ln \left(\left(1-p_{\mathrm{ma}}\right)\left(1-p_{\mathrm{fa}}\right) / p_{\mathrm{ma}} p_{\mathrm{fa}}\right)>0$. Therefore, the result of the forward difference is as follows:

$$
k \leq \frac{\ln \left(c_{\mathrm{fa}} / c_{\mathrm{ma}}\right)-M \ln \left(p_{\mathrm{ma}} /\left(1-p_{\mathrm{fa}}\right)\right)}{\ln \left(\left(1-p_{\mathrm{ma}}\right)\left(1-p_{\mathrm{fa}}\right) / p_{\mathrm{ma}} p_{\mathrm{fa}}\right)}+1
$$

The results of (3) are as follows:

$$
\begin{aligned}
& k \geq N_{l}, \quad k \in[1, M-1], \\
& k \leq N_{l}+1, \quad k \in[2, M],
\end{aligned}
$$

where the notation $N_{l}$ denotes the ONS, which is given as follows:

$$
N_{l}=\frac{\ln \left(c_{\mathrm{fa}} /\left(1-c_{\mathrm{fa}}\right)\right)+M \ln \left(\left(1-p_{\mathrm{fa}}\right) / p_{\mathrm{ma}}\right)}{\ln \left(\left(1-p_{\mathrm{fa}}\right) / p_{\mathrm{ma}}\right)+\ln \left(\left(1-p_{\mathrm{ma}}\right) / p_{\mathrm{fa}}\right)} .
$$

Then the solutions of (2) can be divided into two situations: namely, $N_{l}$ is an integer or not an integer:

$$
= \begin{cases}N_{l} \text { or } N_{l}+1, & \text { if } N_{l} \text { is an integer } \\ \max \left\{m \in Z \mid m<N_{l}+1\right\}, & \text { if } N_{l} \text { is not an integer. }\end{cases}
$$

The EFAR denotes the comprehensive effect of FAR and MAR. In what follows, a case study was presented, namely, the relationship between FAR, MAR, and EFAR. We assume that the TNS $M$ is $10, c_{\mathrm{fa}}$ is 0.5 , and false alarm rate $p_{\mathrm{fa}}$ and the missed alarm rate $p_{\mathrm{ma}}$ are 0.3 and 0.4 , respectively. Figure 1 shows the FAR, MAR, and EFAR as a function of SNS. With the increase of SNS, the FAR decreases (Figure 1(a)) and MAR increases (Figure 1(b)). For a fixed TNS, the feasible range of SNS should be an integer which is greater than or equal to 1 and less than or equal to TNS $M$. The more the number of sensors chosen is, the smaller the FAR and the bigger the MAR of the MAS would be. The reason why MAR increases is that the probability of more sensors in alarm state is smaller than less sensors in alarm state. If bigger SNS is chosen, the probability of MA would increase. The tradeoffs of FAR and MAR are substituted by EFAR which shows concave upward in the feasible range (Figure 1(c)). The minimum value of EFAR is 0.16 (Figure $1(\mathrm{c})$ ), at $\mathrm{SNS}=5$, which is much less than the EFAR at $\mathrm{SNS}=1$ or $\mathrm{SNS}=10$.

\section{The Influences of Parameters}

There are four parameters in the expression of ONS (5), namely, the weight $c_{\mathrm{fa}}$, the false alarm rate of each sensor $p_{\text {fa }}$, the missed alarm rate of each sensor $p_{\text {ma }}$, and the total number of sensors $M$. The factors affecting the EFAR can be divided by three aspects: weight and TNS and the rates including $p_{\mathrm{fa}}$ and $p_{\mathrm{ma}}$.

With the constant TNS, $p_{\mathrm{fa}}$, and $p_{\mathrm{ma}}$, the relationship between the EFAR and the weight $c_{\mathrm{fa}}$ can be obtained. An example is given with TNS $=10, p_{\mathrm{fa}}=0.4$, and $p_{\mathrm{ma}}=0.4$, and the ONS and the minimum EFAR (MiEFAR) as the functions of $c_{\mathrm{fa}}$ are shown in Figure 2. Figure 2(a) shows that the ONS increases with the increase of weight $c_{\mathrm{fa}}$. The scope of $c_{\mathrm{fa}}$ covers from 0 to 1 , exclusively indicating that more attention should be paid to the FAR. The increasing ONS means that if the output of the MAS is alarm state, more and more SNS would be taken with the increase of $c_{\mathrm{fa}}$. Figure 3(b) illustrates the MiEFAR as a function of the weight $c_{\mathrm{fa}}$. With the increase of $c_{\mathrm{fa}}$ from 0.1 to 0.9 , the MiEFAR increases initially but decreases later (Figure 2(b)). The FAR and MAR of the MAS are equally important at $c_{\mathrm{fa}}=0.5$. The maximum value of MiEFAR shows that it is harder to reduce the FAR and the MAR simultaneously when the FAR and the MAR are equally important. Fortunately, in many real-life applications, the weights of FAR and MAR are often not equal [19]. If $c_{\mathrm{fa}}=$ 0.1 or $c_{\mathrm{fa}}=0.9$, the EFAR is mainly determined by only one parameter, and the reduction of that parameter is relatively easier by adjusting the SNS. In particular, at $c_{\mathrm{fa}}=0.1$, the EFAR reduces to be nearly a third of that at $c_{\mathrm{fa}}=0.5$.

Figure 3 shows the MiEFAR as a function of TNS. With the increase of TNS, the MiEFAR decreases. In spite of the changes in $c_{\mathrm{fa}}, p_{\mathrm{fa}}$, and $p_{\mathrm{ma}}$, the MiEFAR decreases with the increase of TNS, which indicates the usefulness of increasing TNS for the MAS. The differences between the weights $c_{\mathrm{fa}}=$ 0.1 and 0.5 show that the nonequal attention paid to FAR and MAR can obtain faster decrease of MiEFAR. The reason is that smaller MAR is more efficient to reduce the EFAR when 


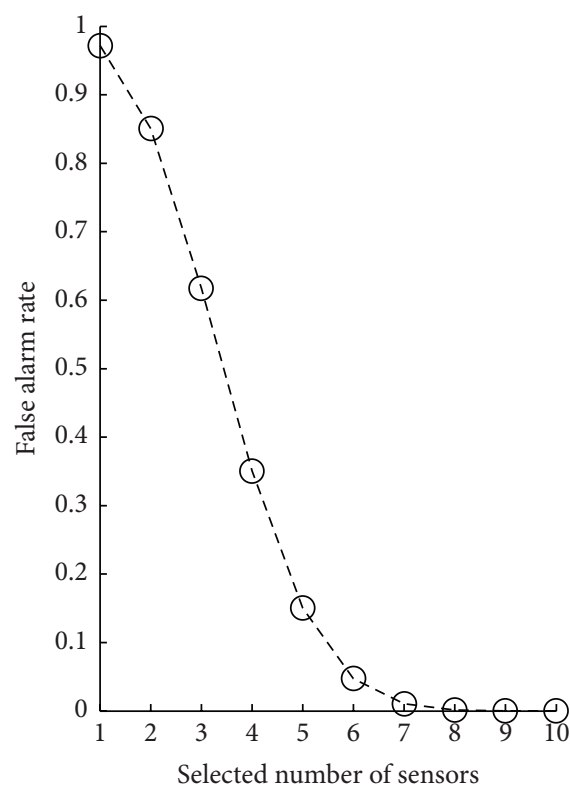

(a)

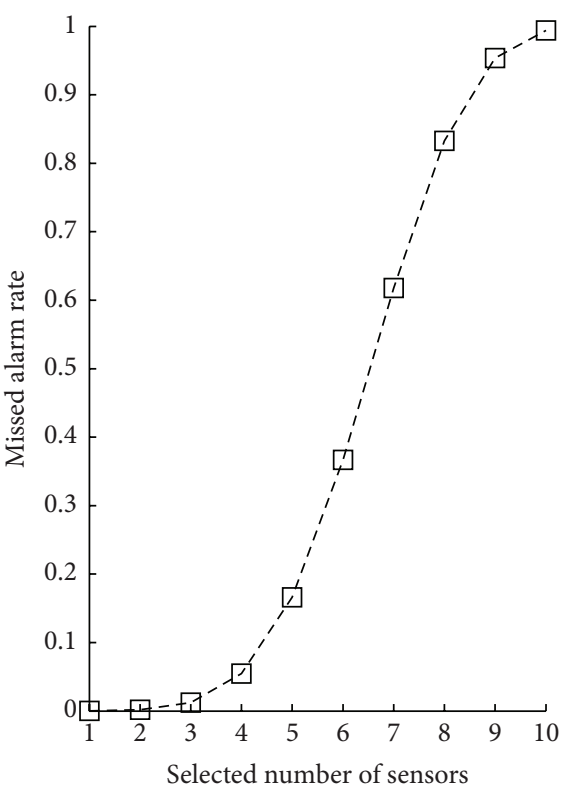

(b)

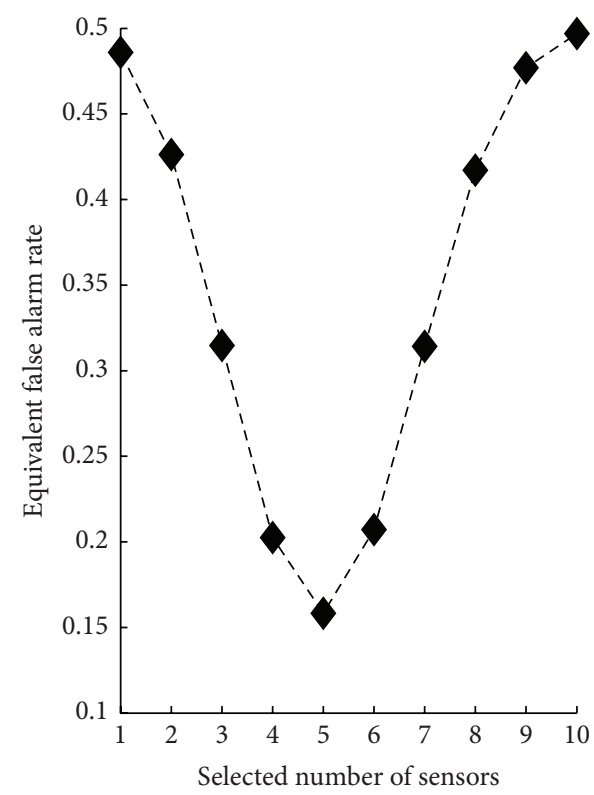

(c)

FIGURE 1: False alarm rate (FAR), missed alarm rate (MAR), and equivalent false alarm rate (EFAR) as a function of selected number of sensors (SNS). (a) The curve of false alarm rate (FAR); (b) the curve of missed alarm rate (MAR); (c) the curve of equivalent alarm rate (EFAR).

$c_{\mathrm{fa}}=0.1$, although it may cause the increase of FAR. However, the efficiency to reduce EFAR is equal for MAR or FAR when $c_{\mathrm{fa}}=0.5$.

Figure 4 shows the MiEFAR as functions of $p_{\mathrm{fa}}$ and $p_{\mathrm{ma}}$. With the increase of either $p_{\mathrm{fa}}$ or $p_{\mathrm{ma}}$, the MiEFAR will increase. The parameters $p_{\mathrm{fa}}$ and $p_{\mathrm{ma}}$ denote the performance of a single sensor in the sensor array, and lower value means better performance. The curve clusters in Figure 4 illustrate that if the performance of a single sensor is better (i.e., smaller $p_{\mathrm{fa}}$ or $p_{\mathrm{ma}}$ ), the sensor array will have a better performance.

\section{Experimental Results on Gas Sensor Array}

The sensor array, which was placed in a $20 \mathrm{~L}$ volume test chamber, was composed of ten metal oxide semiconductor gas sensors TGS 2620. The sensor array resistances were acquired by a half-bridge configuration and then were collected by a multifunction switch/measure unit 34980A via electrical interface on the chamber.

A computer-supervised continuous flow system was built to generate the desired gas concentrations in a highly reproducible way. The experimental setup was shown in Figure 5. 


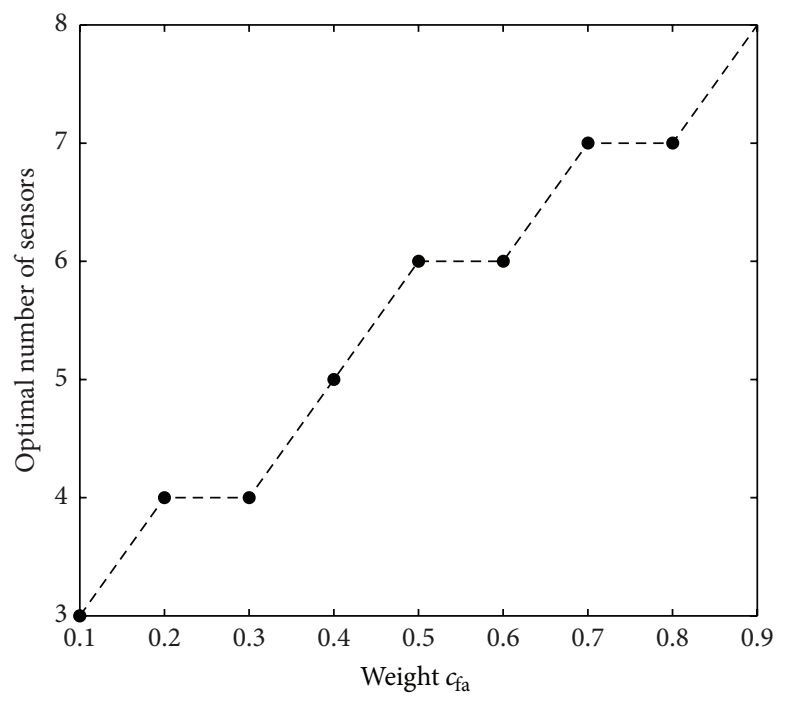

(a) $p_{\mathrm{fa}}=0.4, p_{\mathrm{ma}}=0.4$, and $M=10$

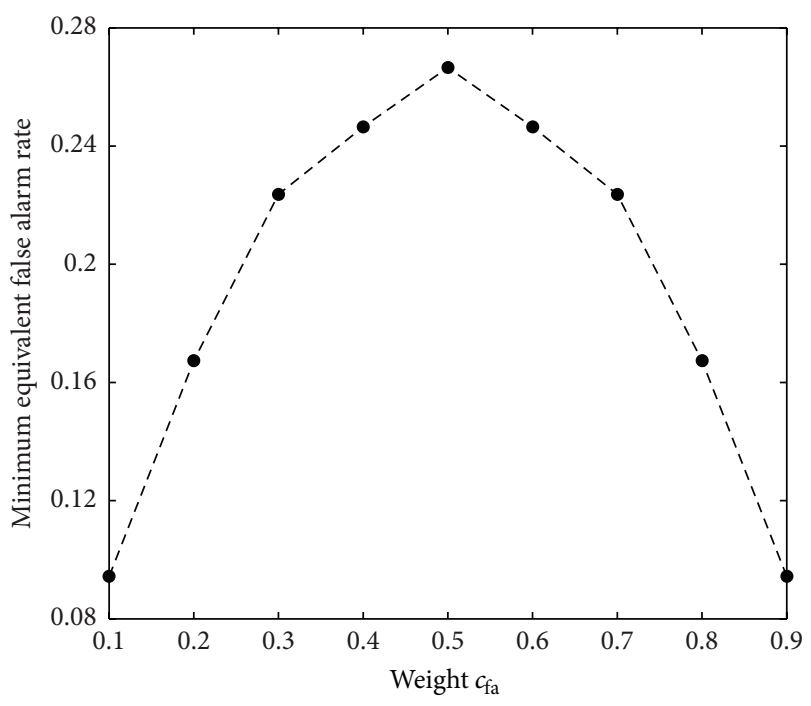

(b) $p_{\mathrm{fa}}=0.4, p_{\mathrm{ma}}=0.4$, and $M=10$

FIGURE 2: Optimal number of sensors (ONS) and minimum equivalent false alarm rate (MiEFAR) as a function of weight $c_{\mathrm{fa}}$. (a) Optimal number of sensors (ONS); (b) minimum equivalent false alarm rate (MiEFAR).

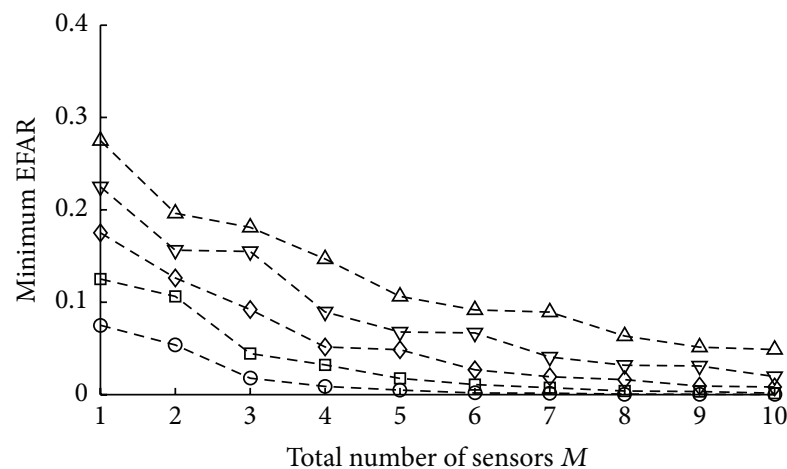

$-\ominus-p_{\mathrm{ma}}=0.05$

一日一 0.15

$-\diamond-0.25$

(a) $c_{\mathrm{fa}}=0.5, p_{\mathrm{fa}}=0.1$

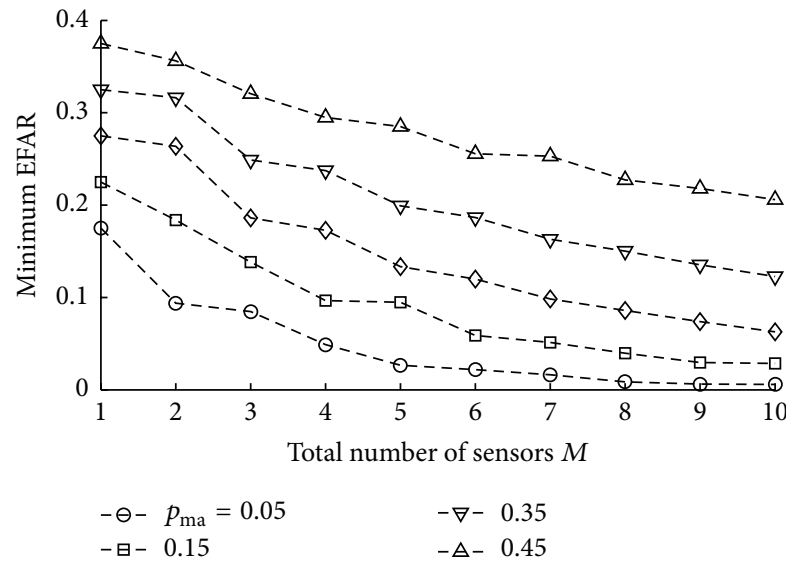

(c) $c_{\mathrm{fa}}=0.5, p_{\mathrm{fa}}=0.3$

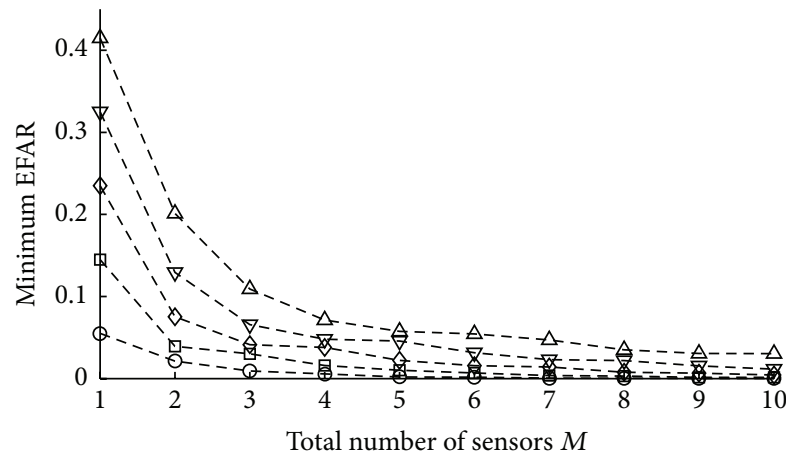

$-\ominus-p_{\mathrm{ma}}=0.05 \quad-\nabla-0.35$

-日一 0.15

-A- 0.45

$-\diamond-0.25$

(b) $c_{\mathrm{fa}}=0.1, p_{\mathrm{fa}}=0.1$

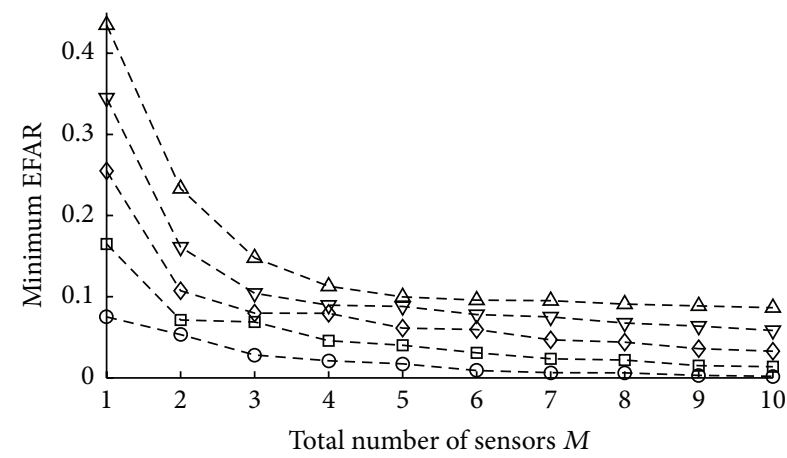

$$
\begin{array}{ll}
-\ominus-p_{\mathrm{ma}}=0.05 & -\nabla-0.35 \\
- \text { - } 0.15 & -\Delta-0.45
\end{array}
$$$$
-\diamond-0.25
$$

(d) $c_{\mathrm{fa}}=0.1, p_{\mathrm{fa}}=0.3$

FIGURE 3: Minimum equivalent false alarm rate (MiEFAR) as a function of total number of sensors (TNS) $M$ : (a) $c_{\mathrm{fa}}=0.5$, $p_{\mathrm{fa}}=0.1 ;(\mathrm{b})$ $c_{\mathrm{fa}}=0.1, p_{\mathrm{fa}}=0.1 ;(\mathrm{c}) c_{\mathrm{fa}}=0.5, p_{\mathrm{fa}}=0.3 ;(\mathrm{d}) c_{\mathrm{fa}}=0.1, p_{\mathrm{fa}}=0.3$. 


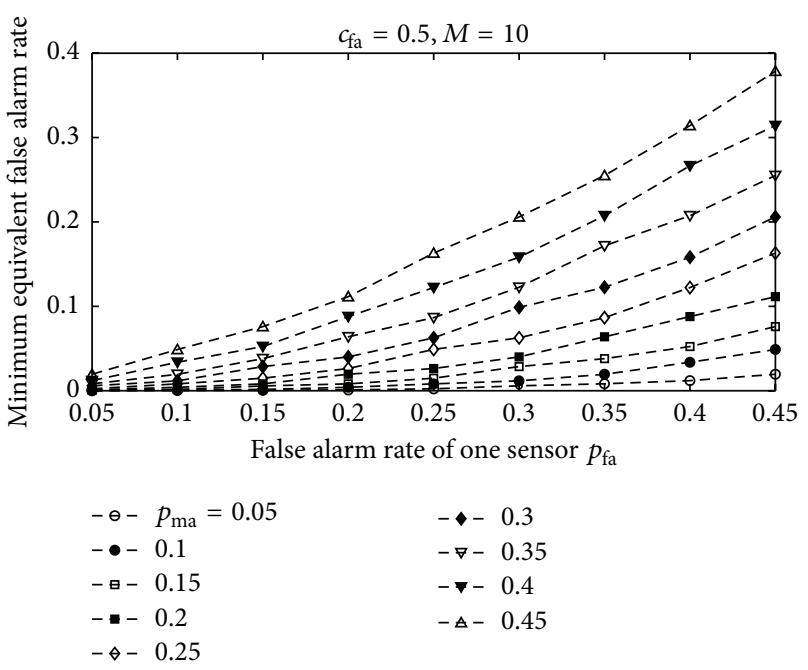

FIgURE 4: The curves of minimum equivalent false alarm rate (MiEFAR) as a function of $p_{\mathrm{fa}}$ when $p_{\mathrm{ma}}$ increases from 0.05 to 0.45 at a step of 0.05 .

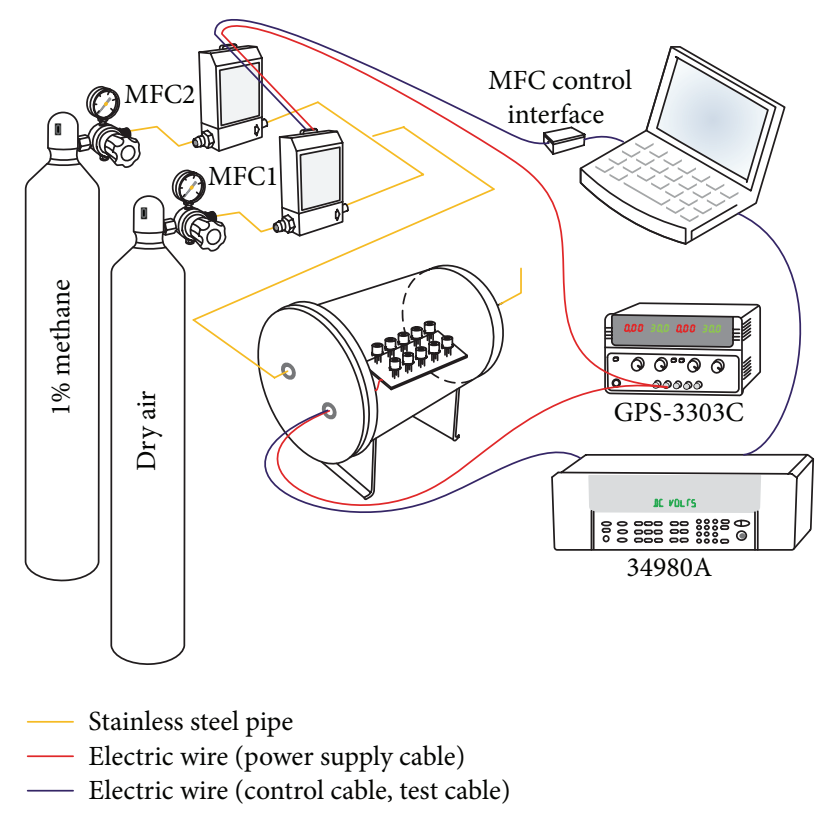

FIGURE 5: The experimental setup: ten metal oxide semiconductor gas sensors TGS 2620 were installed in the middle of the test chamber.

The gas cylinders provided standard dry gases, and in this paper the target gas was methane and the carrier gas was dry air.

A written-in-house LABVIEW program running on a PC platform was used for controlling the mass flow controller (MFC) and collecting data from 34980A. The test concentration was set to $5000 \mathrm{ppm}$, since this concentration was usually treated as the forecasting concentration of methane explosion. The gas flow was set to $300 \mathrm{~mL} / \mathrm{min}$ and kept constant. The measurement was divided into two steps. The very first step is to estimate the parameters $p_{\mathrm{fa}}$ and $p_{\mathrm{ma}}$ via averaging each sensor's false alarm rate and missed alarm rate.
For a single sensor, the false alarm rate $p_{\mathrm{fa}}$ is evaluated by the number of negatives incorrectly classified divided by the total negatives and the missed alarm rate $p_{\mathrm{ma}}$ is calculated by the number of positives incorrectly classified divided by the total positives. Second, using the theoretical values of (15) and (16), the ONS of the sensor array are, respectively, obtained by the thresholds (range from $4800 \mathrm{ppm}$ to $5200 \mathrm{ppm}$ ).

The EFARs were tallied when the TNS changes from 1 to 10. The ONS method presented in this paper was compared with the following common monitoring strategies. (a) All negative resulting negative (ANRN) means that if the outputs of all sensors are in nonalarm state, the output of the MAS is in nonalarm state, which is equivalent to $n=1$. This strategy pays more attention to reducing the MAR and may result in high FAR. (b) All positive resulting positive (APRP) means that if the outputs of all sensors are in alarm state, the output of MAS is in alarm state, which is corresponding to $n=M$. This strategy pays more attention to reducing the FAR and may result in high MAR. (c) The average strategy (AS) means that the SNS is the median of the TNS of the sensor array, which is corresponding to $n=(M+1) / 2$ when TNS is an odd. There are two detailed strategies of AS, namely, AS1 and AS2. AS1 is the strategy that if the TNS is an even number, the SNS takes middle value and AS2 takes the middle value plus one. This strategy takes both FAR and MAR into account equally.

Our objective is to assess the current performance of the MAS with the four common monitoring strategies, namely, ANRN, APRP, AS1, and AS2, and the provided ONS strategy. To get a summarised illustration on the performance of ONS, ROC curves and AUC results are used based on some different thresholds. Then ten ROC curves are plotted using the thresholds settings (range from $4800 \mathrm{ppm}$ to $5200 \mathrm{ppm}$ ) in Figure 6(a) and five ROC curves are plotted in Figure 6(c), where the solid line is for the ONS, the dash-dotted line is for the ANRN, the dash-dot-dotted line is produced by the APRP, the dash line is for the AS1, and the dot line is for the AS2. Based on the theoretical values of (15) and (16), the optimised parameters of ONS vary across different thresholds, while the other strategies do not. This illustrates that the true positive rate of the ONS method increases much faster than those of the other four methods in terms of this experiment. Therefore, the AUC of the ONS method is the highest among the five strategies. A higher AUC value indicates a better classification performance. Figure 6(c) graphically proves that the ONS method presents the best performance, and it is shown in Figure 6(d) that the ONS method has the highest AUC value (0.9631).

\section{Conclusions}

The development of sensors technologies makes higher credibility possible by increasing the number of sensors in the MAS. Credibility is one of the most important parameters for the MAS, especially in high-risk industries. Using a sensor array is one of the potential efficient approaches to enhance the credibility but should be along with an efficient strategy for monitoring. This work provides an analytical 


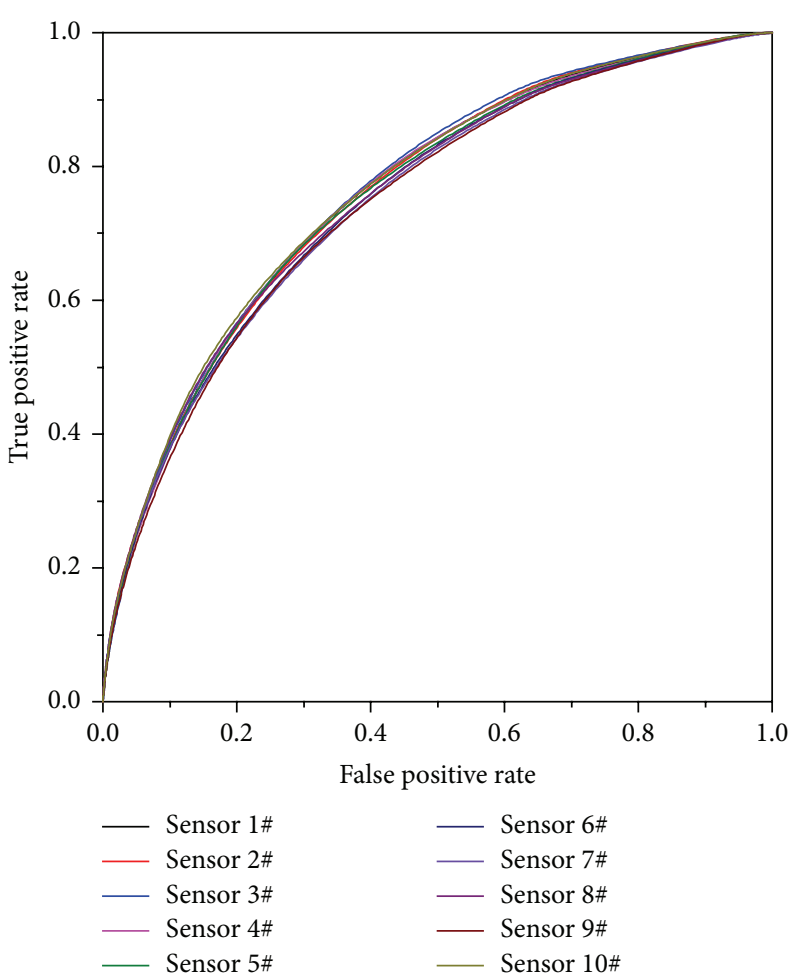

(a)

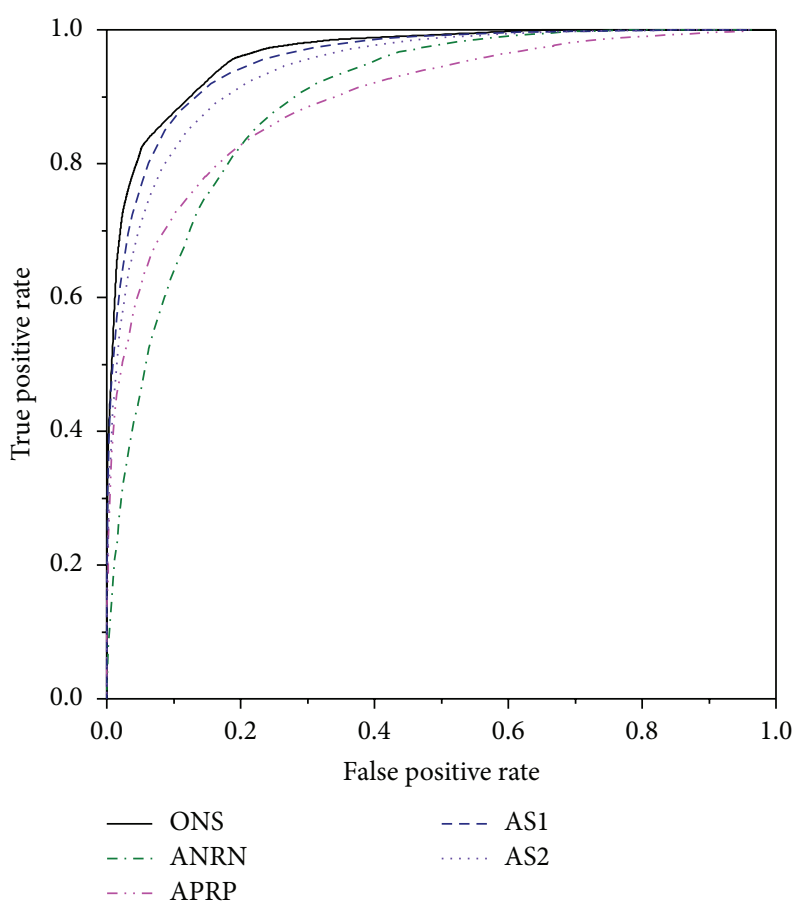

(c)

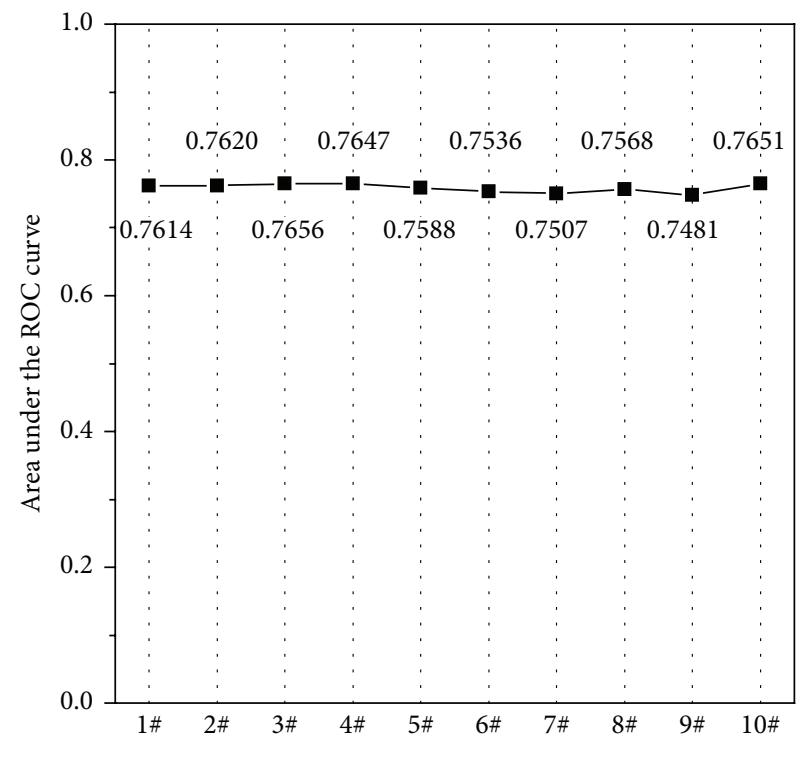

(b)

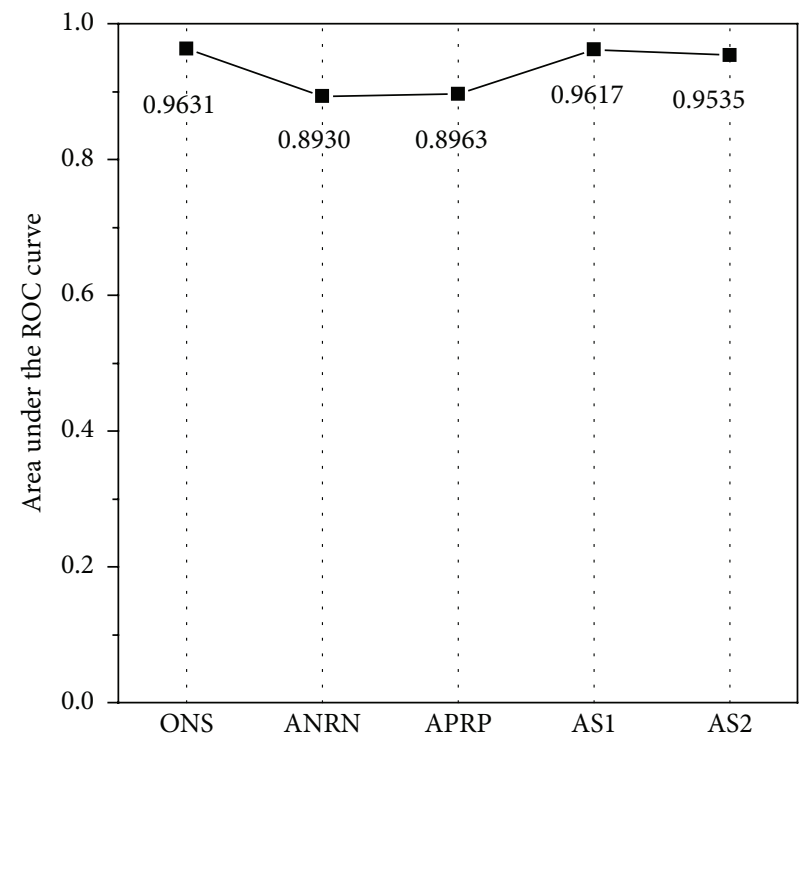

(d)

FIgURE 6: The ROC curves and the area under the ROC curve. (a) The ROC curves of ten sensors (sensor 1\# $\sim$ sensor 10\#); (b) the area under the ROC curve of ten sensors (sensor 1\# sensor 10\#); (c) the ROC curves of five strategies; (d) the area under the ROC curve of the five strategies. 
method to select the ONS for alarm in the MAS based on four parameters, namely, false alarm rate of one sensor $p_{\mathrm{fa}}$, missed alarm rate of one sensor $p_{\mathrm{ma}}$, weight $c_{\mathrm{fa}}$, and TNS $M$. Feasible ONS of the monitoring strategy will release the potential of the MAS with the sensor array to improve the credibility. The results show the effectiveness of credibility enhancement in the MAS. The weight $c_{\mathrm{fa}}$ has the effect of adjusting the ONS, and when more attention is paid for FAR, the bigger ONS will be taken if the TNS is constant. With the increase of TNS, the smaller EFAR could be obtained which shows the effectiveness of using larger number of sensors and the proposed approach for the situation demanding higher credibility. An experimental setup, which contained ten gas sensors, was developed. The ROC curves and the AUC values of ANRN, APRP, AS1, and AS2 and the proposed approach show the effectiveness of the method. The results of this work have the potential application in providing the ONS of a monitoring sensor array, especially in the high-risk industry.

\section{Abbreviations}

ANRN: All negative resulting negative

APRP: All positive resulting positive

AS: $\quad$ Average strategy

AUC: Area under an ROC curve

EFAR: Effective false alarm rate

FA: $\quad$ False alarm

FAR: $\quad$ False alarm rate $p_{\mathrm{fa}}$

MA: $\quad$ Missed alarm

MAR: $\quad$ Missed alarm rate $p_{\mathrm{ma}}$

MAS: $\quad$ Monitor and alarm system

MFC: $\quad$ Mass flow controller

MiEFAR: Minimum effective false alarm rate

ONS: Optimal number of sensors

ppm: $\quad$ Parts per million

ROC: Receiver operating characteristic curve

SNS: $\quad$ Selected number of sensors

TNS: Total number of sensors.

\section{Conflict of Interests}

The authors declare that there is no conflict of interests regarding the publication of this paper.

\section{Acknowledgments}

The authors would like to thank the National Natural Science Foundation of China (nos. 61131004 and 61274076) and the National High Technology Research and Development Program of China (nos. 2006AA040102 and 2006AA040106).

\section{References}

[1] J. Dong, D. Zhuang, Y. Huang, and J. Fu, "Advances in multisensor data fusion: algorithms and applications," Sensors, vol. 9, no. 10, pp. 7771-7784, 2009.

[2] F. Wang, Y. Song, Z. Zhang, and W. Chen, "Structure analysis and decoupling research of a novel flexible tactile sensor array," Journal of Sensors, vol. 2015, Article ID 476403, 10 pages, 2015.
[3] N. Queralto, A. N. Berliner, B. Goldsmith, R. Martino, P. Rhodes, and S. H. Lim, "Detecting cancer by breath volatile organic compound analysis: a review of array-based sensors," Journal of Breath Research, vol. 8, no. 2, Article ID 027112, 2014.

[4] T. Tanantong, E. Nantajeewarawat, and S. Thiemjarus, "False alarm reduction in BSN-based cardiac monitoring using signal quality and activity type information," Sensors, vol. 15, no. 2, pp. 3952-3974, 2015.

[5] C. Pohl and J. L. van Genderen, "Multisensor image fusion in remote sensing: concepts, methods and applications," International Journal of Remote Sensing, vol. 19, no. 5, pp. 823-854, 1998.

[6] E. Zervas, A. Mpimpoudis, C. Anagnostopoulos, O. Sekkas, and S. Hadjiefthymiades, "Multisensor data fusion for fire detection," Information Fusion, vol. 12, no. 3, pp. 150-159, 2011.

[7] W. Liu, J. Wei, M. Liang, Y. Cao, and I. Hwang, "Multi-sensor fusion and fault detection using hybrid estimation for air traffic surveillance," IEEE Transactions on Aerospace and Electronic Systems, vol. 49, no. 4, pp. 2323-2339, 2013.

[8] M. S. Safizadeh and S. K. Latifi, "Using multi-sensor data fusion for vibration fault diagnosis of rolling element bearings by accelerometer and load cell," Information Fusion, vol. 18, no. 1, pp. 1-8, 2014.

[9] S. Coraluppi and C. Carthel, "Recursive track fusion for multisensor surveillance," Information Fusion, vol. 5, no. 1, pp. 23-33, 2004.

[10] B. Hermans and R. Puers, "A portable multi-sensor data-logger for medical surveillance in harsh environments," Sensors and Actuators A: Physical, vol. 123-124, pp. 423-429, 2005.

[11] W. I. S. Galpothdeniya, K. S. McCarter, S. L. De Rooy et al., "Ionic liquid-based optoelectronic sensor arrays for chemical detection," RSC Advances, vol. 4, no. 14, pp. 7225-7234, 2014.

[12] T. Fawcett, "An introduction to ROC analysis," Pattern Recognition Letters, vol. 27, no. 8, pp. 861-874, 2006.

[13] T. Fawcett, "ROC graphs with instance-varying costs," Pattern Recognition Letters, vol. 27, no. 8, pp. 882-891, 2006.

[14] X. Ding, Y. Li, A. Belatreche, and L. P. Maguire, "An experimental evaluation of novelty detection methods," Neurocomputing, vol. 135, pp. 313-327, 2014.

[15] A. P. Bradley, "The use of the area under the ROC curve in the evaluation of machine learning algorithms," Pattern Recognition, vol. 30, no. 7, pp. 1145-1159, 1997.

[16] N. R. Cook, "Use and misuse of the receiver operating characteristic curve in risk prediction," Circulation, vol. 115, no. 7, pp. 928-935, 2007.

[17] A. Yan, W. Wang, C. Zhang, and H. Zhao, "A fault prediction method that uses improved case-based reasoning to continuously predict the status of a shaft furnace," Information Sciences, vol. 259, pp. 269-281, 2014.

[18] F. Yang, D. Xiao, and S. L. Shah, "Optimal sensor location design for reliable fault detection in presence of false alarms," Sensors, vol. 9, no. 11, pp. 8579-8592, 2009.

[19] W. J. Koshak, M. F. Stewart, H. J. Christian, J. W. Bergstrom, J. M. Hall, and R. J. Solakiewicz, "Laboratory calibration of the optical transient detector and the lightning imaging sensor," Journal of Atmospheric and Oceanic Technology, vol. 17, no. 7, pp. 905-915, 2000.

[20] J. Ahnlund, T. Bergquist, and L. Spaanenburg, "Rule-based reduction of alarm signals in industrial control," Journal of Intelligent \& Fuzzy Systems, vol. 14, no. 2, pp. 73-84, 2003.

[21] J. Liu, K. W. Lim, W. K. Ho, K. C. Tan, R. Srinivasan, and A. Tay, "The intelligent alarm management system," IEEE Software, vol. 20, no. 2, pp. 66-71, 2003. 
[22] R. Brooks, R. Thorpe, and J. Wilson, "A new method for defining and managing process alarms and for correcting process operation when an alarm occurs," Journal of Hazardous Materials, vol. 115, no. 1-3, pp. 169-174, 2004.

[23] J. Xu, J. Wang, I. Izadi, and T. Chen, "Performance assessment and design for univariate alarm systems based on FAR, MAR, and AAD," IEEE Transactions on Automation Science and Engineering, vol. 9, no. 2, pp. 296-307, 2012. 


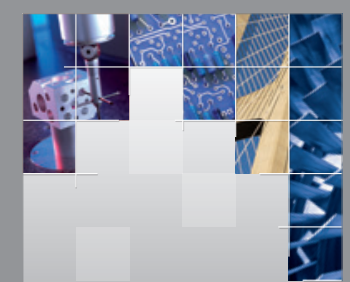

\section{Enfincering}
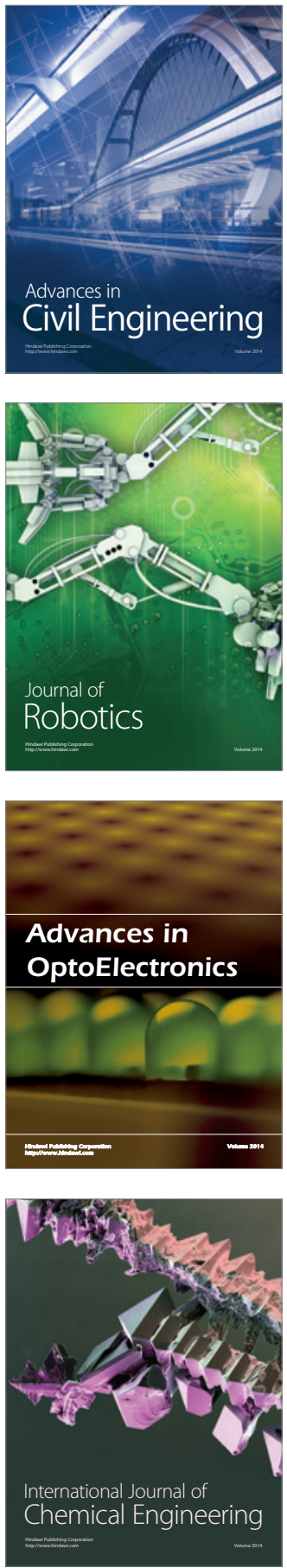

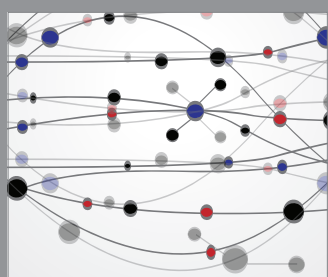

The Scientific World Journal

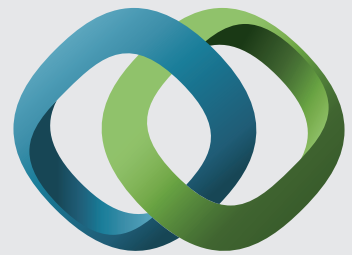

\section{Hindawi}

Submit your manuscripts at

http://www.hindawi.com
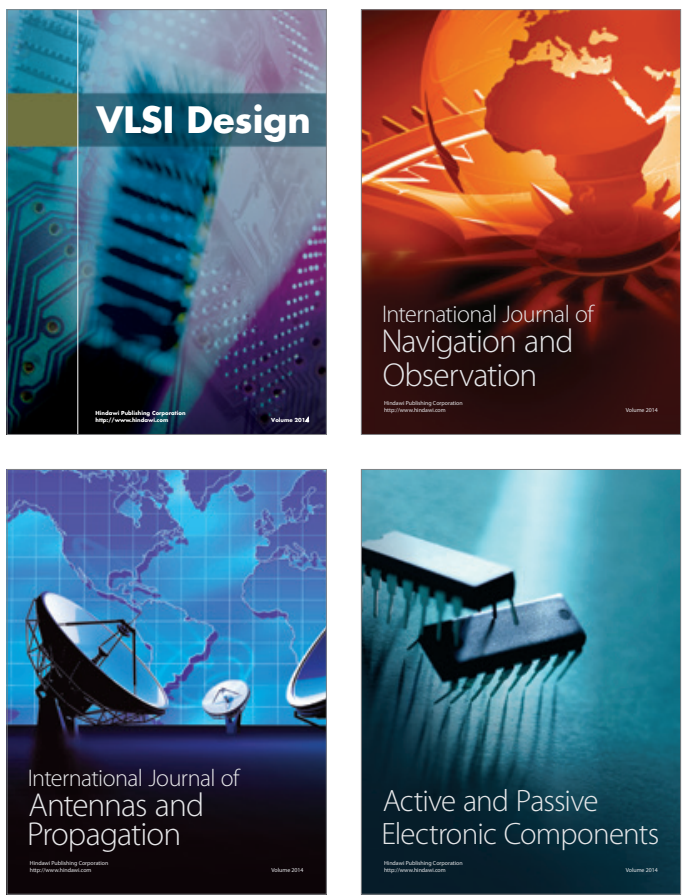
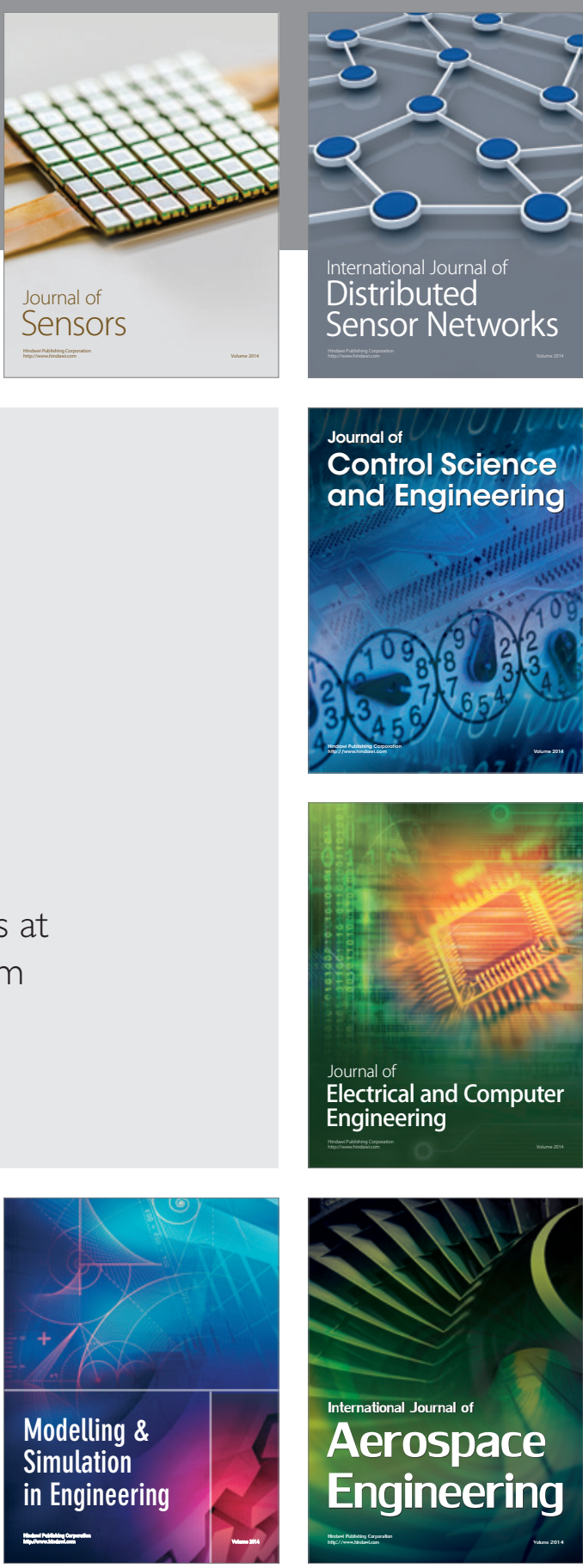

International Journal of

Distributed

Sensor Networks

Journal of

Control Science

and Engineering
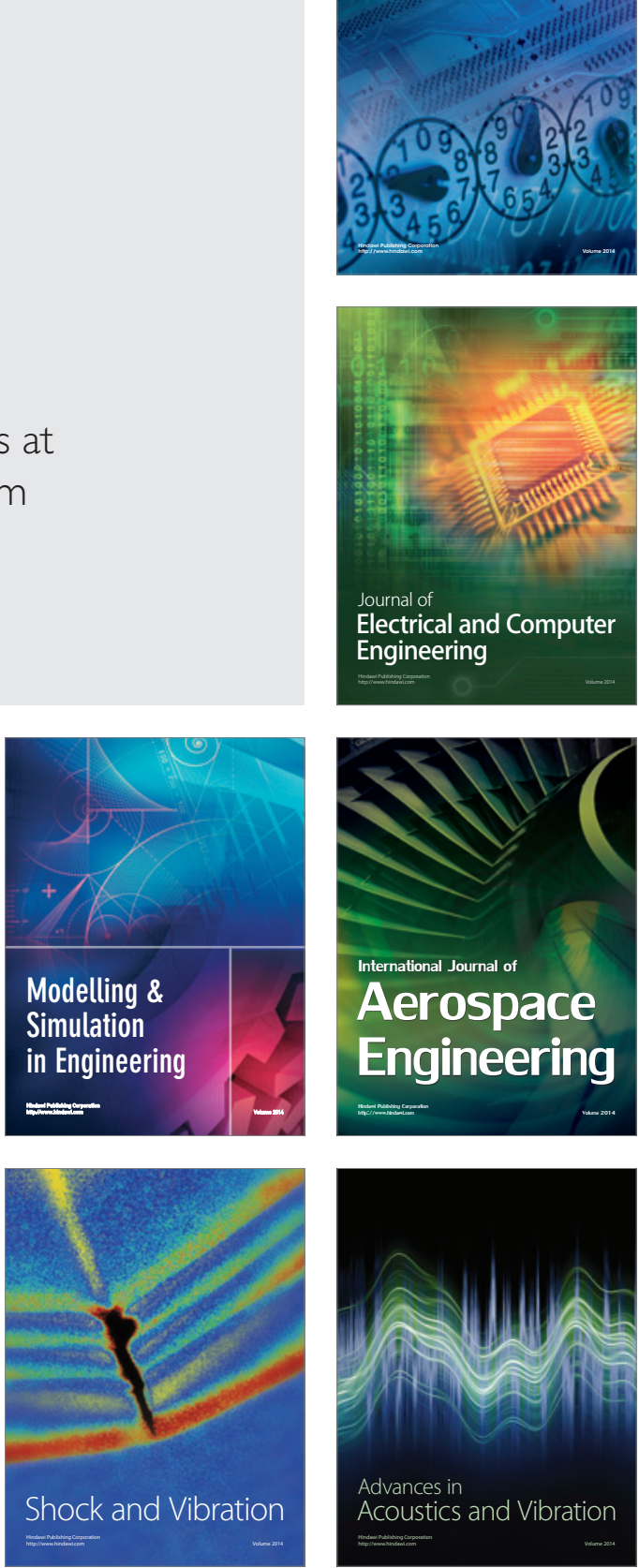